\section{Host-induced epidemic spread of the cholera bacterium}

\author{
D. Scott Merrell ${ }^{\star} \dagger$, Susan M. Butler ${ }^{\star}$, Firdausi Qadri $\ddagger$, Nadia A. Dolganov $\S$, \\ Ahsfaqul Alam $\neq$, Mitchell B. Cohen\|, Stephen B. Calderwood ๆ, \\ Gary K. Schoolnik $\$$ \& Andrew Camilli*
}

* Department of Molecular Biology and Microbiology, Tufts University School of Medicine, Boston, Massachusetts 02111, USA

$\ddagger$ International Centre for Diarrhoeal Disease Research, Mohakhali, Dhaka 1212, Bangladesh

$\$$ Stanford Medical School, Beckman Center, Room 241, Stanford, California 94305, USA

|| Children's Hospital Medical Center, Cincinnati, Ohio 45229, USA

II Division of Infectious Diseases, Massachusetts General Hospital, Boston,

Massachusetts 02114, and Harvard Medical School, Boston, Massachusetts 02115, USA

The factors that enhance the transmission of pathogens during epidemic spread are ill defined. Water-borne spread of the diarrhoeal disease cholera occurs rapidly in nature, whereas infection of human volunteers with bacteria grown in vitro is difficult in the absence of stomach acid buffering ${ }^{1}$. It is unclear, however, whether stomach acidity is a principal factor contributing to epidemic spread ${ }^{2}$. Here we report that characterization of Vibrio cholerae from human stools supports a model whereby human colonization creates a hyperinfectious bacterial state that is maintained after dissemination and that may contribute to epidemic spread of cholera. Transcriptional profiling of $V$. cholerae from stool samples revealed a unique physiological and behavioural state characterized by high expression levels of genes required for nutrient acquisition and motility, and low expression levels of genes required for bacterial chemotaxis.

Frequent cholera outbreaks occur in Dhaka, Bangladesh, providing the potential to study the bacterium within its natural setting. We collected stool samples from patients at the International Centre for Diarrhoeal Disease Research (ICDDR), Bangladesh, and performed serological analysis to identify stools that were positive for $V$. cholerae $\mathrm{O} 1$ Inaba $\mathrm{El}$ Tor. These were immediately used in murine infection studies. A strain grown in vitro (DSM-V984) was mixed with stool $V$. cholerae and the mixture was used to inoculate 3-5day-old Swiss Webster mice by gavage. DSM-V984 is an O1 Inaba El Tor strain that was isolated the previous year at the ICDDR, and is marked by deletion of the lacZ gene to allow for enumeration of stool $\left(\mathrm{LacZ}^{+}\right)$and in vitro-grown (DSM-V984; $\mathrm{LacZ}^{-}$) bacteria. Mice were euthanized after $20-24 \mathrm{~h}$, and bacteria were recovered by homogenization of the small intestine and plating on medium supplemented with a chromogenic substrate for LacZ. The output ratios of stool-sample $V$. cholerae to DSM-V984 were corrected for variations in the input ratios and represent the competitive indices (CI) of the stool $V$. cholerae. A CI above 1 indicates enhanced infectivity, whereas an index below 1 indicates decreased infectivity. As shown in Fig. 1a, V. cholerae shed from the human gastrointestinal tract (human-shed) showed greatly enhanced infectivity, outcompeting the in vitro-grown strain by as much as 700 -fold. A competitive advantage was consistently observed with samples collected from numerous patients on different days. The competitive advantage was entirely lost, however, when $V$. cholerae was colony purified from a stool sample and cultured in vitro in broth for $18 \mathrm{~h}$ before infection (CI geometric means of 1.1, 1.1 and 1.5 for three independent stool isolates and 8-10 mice per competition), indicating that passage through the human host transiently increased the infection potential of the stool-derived bacteria.

† Present address: Department of Microbiology and Immunology, Stanford School of Medicine, Stanford, California 94305, USA.
To have a significant role in the epidemic spread of cholera, the competitive advantage of stool-derived bacteria would have to persist after dissemination back into the environmental reservoir. We therefore tested whether human-shed $V$. cholerae maintained the hyperinfectious phenotype after incubation in local pond water. Freshly shed $V$. cholerae positive stool samples were diluted into pond water that was free of $V$. cholerae contamination. After incubation at room temperature $\left(29^{\circ} \mathrm{C}\right)$ for $5 \mathrm{~h}$, the diluted samples were mixed with the in vitro-grown competitor strain, and this mixture was used to infect mice as described above. As shown in Fig. $1 \mathrm{~b}$, the hyperinfectious state of the human-shed bacteria remained intact. We propose that human passage of $V$. cholerae enhances subsequent water-borne spread of the cholera bacterium by effectively lowering the infectious dose in secondary individuals.

As the increased infectivity of stool-derived $V$. cholerae was lost after growth in vitro, the phenotype is transiently expressed. Therefore, to characterize the hypervirulent state we conducted transcriptional profiling of human-shed $V$. cholerae using a spotted DNA microarray ${ }^{3}$ containing representations of approximately $87 \%$ of the identified open reading frames (ORFs) of the Bangladeshi O1 El Tor reference strain N16961 (ref. 4). O1 Inaba El Tor positive stool samples were obtained from three patients in the ICDDR, filtered to remove particulate matter, then immediately frozen for later preparation of total RNA. These samples showed the canonical 'rice water' appearance that characterizes cholera stools, contained approximately 100 million $V$. cholerae per millilitre, and showed relatively few contaminating bacteria as assessed by dark-field microscopy and plating 5 . Stool RNA preparations were analysed by agarose gel electrophoresis to ensure RNA integrity and the absence of contaminating eukaryotic RNA. DSM-V999 was isolated from one of the three stool samples, and total RNA was isolated after in vitro growth to stationary phase (growth conditions identical to those used for the growth of DSM-V984 in the competition assays described above). One microgram of RNA from each of the samples was used for complementary DNA synthesis, labelled with Cy5 and hybridized to the microarray in the presence of a Cy3-labelled common reference (exponentially growing O1 Inaba El Tor strain 92A1552). Each sample was hybridized in quadruplicate and the relative fluorescent intensities determined by scanning with an Axon scanner. Data were quantified, normalized and corrected to yield the relative transcript abundance of each gene as an intensity ratio with respect to that of the reference signal. These intensity ratios were then used to identify statistically significant differences
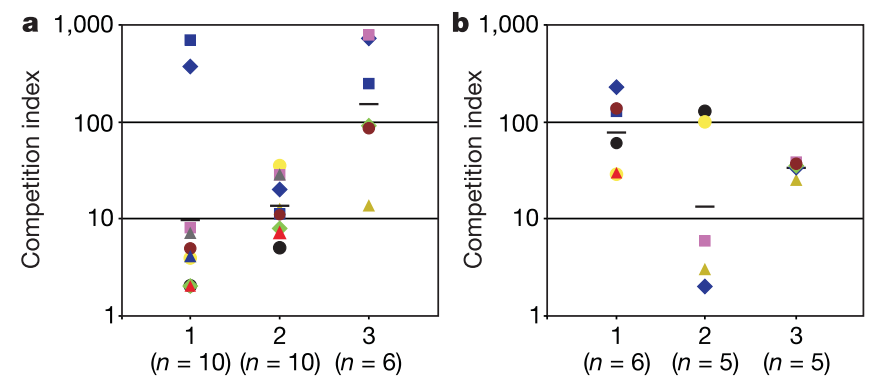

Figure 1 Human-shed $V$. cholerae are hyperinfectious in competition assays in infant mice. a, b. Freshly isolated stool samples from six patients (representing experiments 1, 2 and 3 on the $x$ axes in $\mathbf{a}$ and $\mathbf{b}$ ) were either mixed directly with in vitro-grown DSM-V984 (a), or incubated in local pond water for $5 \mathrm{~h}$ before mixing (b), and then used for intragastric infection of animals. Data points represent the output ratio of stool-derived $V$. cholerae to in vitro-grown competitor strain after correction for deviations in input ratios. Each data point represents the output from a single animal and symbols and colours are used to facilitate visual discrimination of data values. The geometric mean is indicated by a horizontal bar. $n$, total number of animals per experiment. 
in gene expression using the Statistical Analysis for Microarrays (SAM) program ${ }^{6}$. A two-class SAM analysis was conducted using the strain grown in vitro as class I, and each individual stool sample as class II. Genes with statistically significant changes in the level of expression - at least a twofold change-in each patient sample were chosen, and the derived data from individual stool samples were collapsed to identify genes that were differentially regulated in all three samples. According to these criteria, 237 genes were differentially regulated: 44 genes were induced and 193 genes were repressed in human-shed $V$. cholerae (Fig. 2 and Supplementary Information).

The transcriptomes (the complete collection of transcribed RNAs) of the stool-derived $V$. cholerae samples were similar to that of strain DSM-V999 cultured in rich medium, as 3,120 out of 3,357 ORFs examined were expressed at roughly equivalent levels. Notable differences included increased expression of an assortment of genes required for biosynthesis of amino acids, iron uptake systems, ribosomal proteins, and formation of a periplasmic nitrate reductase complex that may allow for respiration under low oxygen tension. This transcriptome is consistent with bacterial growth in an oxygen- and iron-limited host compartment-the conditions encountered in rice-water stools ${ }^{7}$. Combining these observations, we propose that $V$. cholerae departs from a rich growth environment within the small intestine and moves into a nutrient poor lumenal fluid that is rapidly purged. The physiological growth state of the stool bacteria and the capability for rapid growth could contribute to the observed hyperinfectious phenotype, as rapidly growing $V$. cholerae out-compete stationary phase cells (A.C., unpublished data, and J. J. Mekalanos, personal communication). However, hierarchical clustering of stationary phase, exponential phase and human-shed $V$. cholorae arrays revealed that $V$. cholorae isolated from patients cluster no closer to the exponential phase than to the stationary phase arrays, suggesting that on a genome-wide scale, the transcriptome bears traits of both of the growth phases (data not shown).

None of the genes that showed differential regulation were members of the ToxR/TcpP/ToxT virulence gene regulon ${ }^{8-11}$. Genes within this regulon include those for cholera toxin and factors coded for on the Vibrio pathogenicity island, such as the toxin co-regulated pilus. These factors are necessary for successful infection of humans and mice ${ }^{12}$. These results suggest that, before being shed, $V$. cholerae turns off expression of these particular virulence genes as part of a programme for dissemination to the environment and possible transmission to new hosts. These findings also indicate that the increased expression of this virulence regulon is not required for the increased infectivity exhibited by stool bacteria.

Among the genes differentially expressed in the stool samples are VC0028, VC0941, VC0869, VC0051, VC0647, VC0468, VC2350 and VCA0583, all of which were recently identified in a genetic screen for factors required for infection of infant mice ${ }^{13}$. VC0647 (pnp) and VC0468 ( $g s h B)$ were additionally shown to have a role in the ability of $V$. cholerae to mount an acid tolerance response (ATR). In vitro induction of the ATR enhances the infectivity of $V$. cholerae ${ }^{14}$; however, our microarray analysis revealed that other genes known to be induced during the ATR were not differentially regulated in stool samples, suggesting that if ATR has an involvement in the increased infectivity of human-shed V. cholerae, it is through pathways yet to be elucidated.

The most highly induced gene that we identified is one that was not annotated on completion of the $V$. cholerae genome sequence. As our $V$. cholerae microarray was constructed before annotation of the published sequence ${ }^{15}$, it contains sequences of some ORFs that

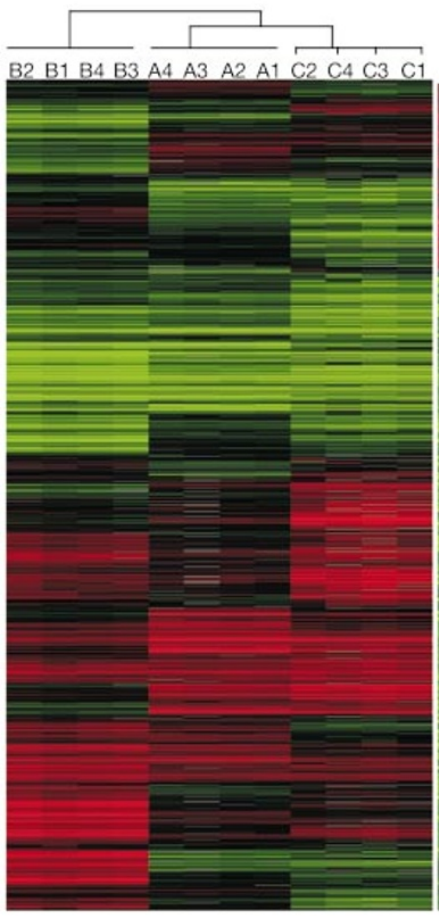

Figure 2 Transcription profile of human-shed $V$. cholerae. Cluster diagram showing the whole-genome expression profile of $V$. cholerae recovered from the stools of three ICDDR patients (left panel). Patient samples are labelled $\mathrm{A}-\mathrm{C}$ and replicate arrays are indicated by the numbers $1-4$. The dendrogram shows tight clustering of technical replicates. The right panel is a cluster diagram of genes showing consistent differential regulation from patients $\mathrm{A}, \mathrm{B}$ and $\mathrm{C}$. Fold changes relative to $\mathrm{V}$. cholerae grown in vitro were calculated

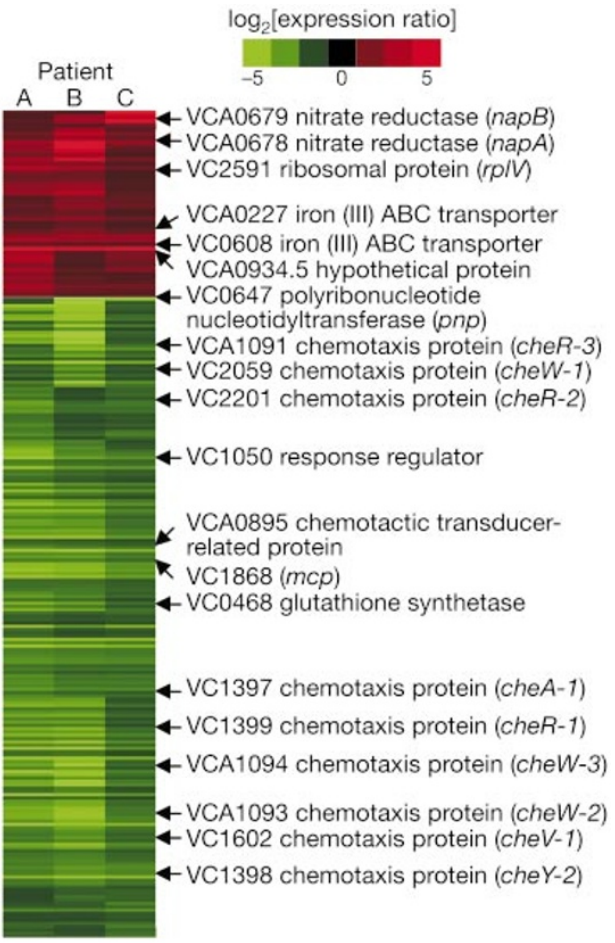

using the average values from the quadruplicate arrays shown on the left, and are schematically represented here. Red indicates a minimum twofold increase in expression; green represents a minimum twofold reduction in expression. Representative genes are listed and their relative location indicated by an arrow. See Supplementary Information for a complete list with relative fold changes. 
did not meet cut-offs imposed during the course of annotation. 'VCA0934.5' (residues 885680-885886) lies in the region between VCA0933 and VCA0935, is transcribed in the same direction as the upstream VCA0933, and partially overlaps the divergently transcribed VCA0934. VCA0934.5 has a predicted strong Shine-Dalgarno sequence and would code for a protein comprising 69 amino acids that shows no significant similarity to any proteins contained in public databases and bears no known protein motifs. Elucidation of the function of this factor will be of great interest and its discovery emphasizes the importance of analysis of DNA sequences that lie outside of the cut-offs commonly used for genomic annotation in bacteria.

A marked number of genes involved in chemotaxis showed altered expression levels: expression of these genes was repressed in the stool-derived $V$. cholerae. The role of chemotaxis in virulence of $V$. cholerae is unclear ${ }^{16,17}$, although several genes required for chemotaxis are known to be crucial for expression of cholera toxin during infection ${ }^{18}$. Notably, these latter genes, including cheA-2, cheY-3 and cheZ, did not show altered expression in the stool samples. Instead, most of the remaining chemotaxis genes were repressed, including genes for all three CheW linker proteins, three out of four CheV linker proteins, all three CheR methyltransferases, and 17 methyl-accepting chemotaxis proteins. Although the stoolderived $V$. cholerae were highly motile as assessed by dark-field and phase-contrast microscopy, many of the gene families that are essential for chemotactic signalling and adaptation to chemoattractant and chemorepellent gradients were repressed. These results suggest that these motile bacteria are non-chemotactic on exiting the human host, and imply that downregulation of the chemotactic response is linked to dissemination from the host and re-entry to the aquatic environment. One possibility is that repression of chemotaxis increases shedding from the gastrointestinal tract. Finally, loss of the chemotactic response in otherwise motile $V$. cholerae was recently shown to markedly increase infectivity in an infant mouse model of cholera ${ }^{18}$. It is possible that the motile but non-chemotactic state combined with the unique physiological state of human-shed $V$. cholerae mediates the observed increase in infectivity. Because this transcriptional profiling provides only a 'snap-shot' of gene expression at the moment of dissemination, an important next step will be the elucidation of the proteome of human-shed $V$. cholerae.

Our findings identify the process by which cholera epidemics may be propagated by the human host, and provide insight into gene expression patterns of the disseminating microorganisms. Passage through the human gastrointestinal tract induces a hyperinfectious state, which is perpetuated even after purging into natural aquatic reservoirs. The fact that the human host not only provides a suitable niche for growth but also prepares $V$. cholerae for infection of additional humans has interesting implications for the study of human-to-human spread of other virulent microorganisms. Perhaps other epidemic pathogens are spread in a manner whereby the agent is made more infectious before or during exit from its primary host. Elucidation of the gene expression profiles of disseminating microorganisms should provide new targets for antimicrobial therapy to disable transmission and for vaccine development to prevent infection.

\section{Methods}

\section{Strains, sample collection and competition assays}

Strain DSM-V984 was differentially marked at the lacZ locus by mobilization of the suicide plasmid pGP704::lac $Z$ and selection on media supplemented with ampicillin and 5-bromo-4-chloro-3-indoyl- $\beta$-D-galactoside (X-gal) as described previously ${ }^{19}$. Patients admitted to the ICDDR, Bangladesh, showing clinical manifestations of cholera had ricewater stools pre-screened by dark-field microscopy to verify the presence of V. cholerae, and by inhibition of dark-field motility using monoclonal antibodies to determine serogroup and serotype. Freshly shed stool samples were collected in sterile beakers, filtered through cheesecloth, and a portion was frozen at $-80^{\circ} \mathrm{C}$ for subsequent RNA preparation. The remainder of the stool sample was used in competition assays. Human studies protocols were reviewed and approved by the Research Review Committee and Ethical Review Committee at the ICDDR, Bangladesh, and by the Institutional Review Board at the Massachusetts General Hospital.

Competition assays were conducted by mixing DSM-V984 grown on LB medium overnight with stool bacteria in a ratio of 1:10 (v/v). A total of $50 \mu \mathrm{l}$ of a 1,000-fold dilution of this mixture, representing $10^{4}-10^{5}$ colony-forming units (c.f.u.), was administered to 3 5 -day-old Swiss Webster mice by gavage as described ${ }^{14}$. After $20-24 \mathrm{~h}$, animals were euthanized, the small intestines removed, homogenized and plated on LB medium supplemented with $\mathrm{X}$-gal to allow for subsequent enumeration of $\mathrm{LacZ}^{+}$and $\mathrm{LacZ}^{-}$ colonies. Outputs ranged from $10^{5}$ to $10^{7}$ c.f.u. per small intestine. Ratios of output bacteria were corrected for deviations in input ratios from 1:1 and represent the competitive index of the strains in question. Competitions with water-incubated stoolderived bacteria were conducted as above with the exception that stool-derived $V$. cholerae were diluted into pond water and were incubated at room temperature for $5 \mathrm{~h}$ before mixing with DSM-V984 and inoculation into mice. The $\mathrm{pH}$ of the two pond water samples was $7.0-7.5$.

\section{Microarray analysis}

The construction of the V. cholerae DNA microarray will be described in greater detail elsewhere. Briefly, after the completion of the genomic sequence of N16961 by the Institute for Genomic Research, but before annotation of the genome, predicted unique ORFs were found using two separate ORF-finding programs, and a portion of each ORF was amplified by polymerase chain reaction (PCR) and spotted onto poly-L-lysine-coated glass slides ${ }^{15}$. The array consists of 5,222 spots representing 3,357 ORFs with an average size of 600 base pairs per PCR product.

Total $V$. cholerae RNA was collected from stool samples and strain DSM-V999 grown overnight in vitro using TRIzol reagent (GIBCO-BRL) as described ${ }^{15}$, with the exception that RNA was collected on an RNeasy column (QIAGEN) immediately after isopropanol precipitation. DNase treatment to remove DNA contamination was conducted before elution from the column. Quantities of RNA were determined by measuring absorbance at $260 / 280 \mathrm{~nm}\left(A_{260} / A_{280}\right)$ and integrity was verified by visualization on a $1 \%$ agarose gel. Equal concentrations of each test RNA and the common reference RNA (exponentially growing 92A1552) were used for reverse transcription reactions that directly incorporate Cy5- and Cy3-labelled nucleotides into the $\mathrm{cDNA}$ as described ${ }^{15}$. Labelling reactions were performed in duplicate on two separate days, resulting in quadruplicate arrays for each strain derived from stool samples or grown in vitro. The hybridizations and data analyses were performed as described in the main text. Control arrays were also hybridized to identify genes potentially affected by the stool-freezing process, and statistically significant changes that matched patient stool sample regulation were eliminated from subsequent analysis (all microarray data are provided at http://genome-www5.stanford.edu/ microarray/SMD/).

Received 18 March; accepted 22 April 2002; doi:10.1038/nature00778.

1. Cash, R. A. et al. Response of man to infection with Vibrio cholerae. I. Clinical, serologic, and bacteriologic responses to a known inoculum. J. Infect. Dis. 129, 45-52 (1974).

2. Gitelson, S. Gastrectomy, achlorhydria and cholera. Israel J. Med. Sci. 7, 663-667 (1971).

3. Schena, M., Shalon, D., Davis, R. W. \& Brown, P. O. Quantitative monitoring of gene expression patterns with a complementary DNA microarray. Science 270, 467-470 (1995).

4. Heidelberg, J. F. et al. DNA sequence of both chromosomes of the cholera pathogen Vibrio cholerae. Nature 406, 477-483 (2000).

5. Freter, R., Smith, H. L. \& Sweeney, F. J. Enumeration of cholera vibrios in faecal samples. J. Infect. Dis. 109, 31-34 (1961).

6. Tusher, V. G., Tibshirani, R. \& Chu, G. Significance analysis of microarrays applied to the ionizing radiation response. Proc. Natl Acad. Sci. USA 98, 5116-5121 (2001).

7. Freter, R., Smith, H. L. \& Sweeney, F. J. An evaluation of intestinal fluids in the pathogenesis of cholera. J. Infect. Dis. 109, 35-42 (1961).

8. Miller, V. L. \& Mekalanos, J. J. Synthesis of cholera toxin is positively regulated at the transcriptional level by toxR. Proc. Natl Acad. Sci. USA 81, 3471-3475 (1984).

9. Carroll, P. A., Tashima, K. T., Rogers, M. B., DiRita, V. J. \& Calderwood, S. B. Phase variation in tcpH modulates expression of the ToxR regulon in Vibrio cholerae. Mol. Microbiol. 25, 1099-1111 (1997).

10. Hase, C. C. \& Mekalanos, J. J. TcpP protein is a positive regulator of virulence gene expression in Vibrio cholerae. Proc. Natl Acad. Sci. USA 95, 730-734 (1998).

11. DiRita, V. J., Parsot, C., Jander, G. \& Mekalanos, J. J. Regulatory cascade controls virulence in Vibrio cholerae. Proc. Natl Acad. Sci. USA 88, 5403-5407 (1991).

12. Herrington, D. A. et al. Toxin, toxin-coregulated pili, and the toxR regulon are essential for Vibrio cholerae pathogenesis in humans. J. Exp. Med. 168, 1487-1492 (1988).

13. Merrell, D. S., Hava, D. L. \& Camilli, A. Identification of novel factors involved in colonization and acid tolerance of Vibrio cholerae. Mol. Microbiol. 43, 1471-1491 (2002).

14. Merrell, D. S. \& Camilli, A. The cadA gene of Vibrio cholerae is induced during infection and plays a role in acid tolerance. Mol. Microbiol. 34, 836-849 (1999).

15. Schoolnik, G. K. et al. Whole genome DNA microarray expression analysis of biofilm development by Vibrio cholerae O1 E1 Tor. Methods Enzymol. 336, 3-18 (2001).

16. Richardson, K. Roles of motility and flagellar structure in pathogenicity of Vibrio cholerae: analysis of motility mutants in three animal models. Infect. Immun. 59, 2727-2736 (1991).

17. Gardel, C. L. \& Mekalanos, J. J. Alterations in Vibrio cholerae motility phenotypes correlate with changes in virulence factor expression. Infect. Immun. 64, 2246-2255 (1996).

18. Lee, S. H., Butler, S. M. \& Camilli, A. Selection for in vivo regulators of bacterial virulence. Proc. Natl Acad. Sci. USA 98, 6889-6894 (2001).

19. Camilli, A. \& Mekalanos, J. J. Use of recombinase gene fusions to identify Vibrio cholerae genes induced during infection. Mol. Microbiol. 18, 671-683 (1995)

Supplementary Information accompanies the paper on Nature's website

(http://www.nature.com/nature). 


\section{Acknowledgements}

We thank E. A. Joyce, M. K. Waldor and A. L. Sonenshein for comments and discussion of the manuscript, C. C. Kim for help with statistical analysis, and S. Falkow and

J. J. Mekalanos for critical discussions. This research was supported by grants from the National Institutes of Health. The ICDDR at Bangladesh is supported by agencies and countries that share its concern for the health problems of the developing world.

Competing interests statement

The authors declare that they have no competing financial interests.

Correspondence and requests for materials should be addressed to A.C. (e-mail: Andrew.Camilli@Tufts.edu).

\section{Origin of GABAergic neurons in the} human neocortex

\section{Kresimir Letinic, Roberto Zoncu \& Pasko Rakic}

Yale University School of Medicine, Section of Neurobiology, New Haven, Connecticut 06510, USA

The mammalian neocortex contains two major classes of neurons, projection and local circuit neurons ${ }^{1-4}$. Projection neurons contain the excitatory neurotransmitter glutamate, while local circuit neurons are inhibitory, containing $\mathrm{GABA}^{2,4}$. The complex function of neocortical circuitry depends on the number and diversity of GABAergic ( $\gamma$-aminobutyric-acid-releasing) local circuit neurons ${ }^{1-3}$. Using retroviral labelling in organotypic slice cultures of the embryonic human forebrain, we demonstrate the existence of two distinct lineages of neocortical GABAergic neurons. One lineage expresses Dlx1/2 and Mash1 transcription factors, represents $65 \%$ of neocortical GABAergic neurons in humans, and originates from Mash1-expressing progenitors of the neocortical ventricular and subventricular zone of the dorsal forebrain. The second lineage, characterized by the expression of Dlx1/2 but not Mash1, forms around 35\% of the GABAergic neurons and originates from the ganglionic eminence of the ventral forebrain. We suggest that modifications in the expression pattern of transcription factors in the forebrain may underlie species-specific programmes for the generation of neocortical local circuit neurons ${ }^{5-11}$ and that distinct lineages of cortical interneurons may be differentially affected in genetic and acquired diseases of the human brain.

Classical studies, as well as modern retroviral lineage analyses, concur that projection neurons, in all species examined, originate in the ventricular zone (VZ) of the dorsal telencephalon and migrate radially across the intermediate zone (IZ) to the overlying cortical plate $e^{6,7,10-14}$. However, recent retroviral lineage and chimaera studies in rodents have demonstrated that local circuit neurons originate in the subcortical ganglionic eminence (GE) of the ventral telencephalon and migrate tangentially through the IZ into the cerebral cortex $^{5-11,15-17}$. Furthermore, mice lacking the transcription factors Dlx or Mash1 that are expressed in the GE but not in the VZ of the dorsal telecephalon lack most cortical GABAergic neurons, confirming their origin from the ventral telecephalon ${ }^{10,18,19}$. Because GABAergic neurons increase in number, complexity and proportion relative to projection neurons during primate evolution $^{1,2,4,20,21}$ and are implicated in a host of neurological disorders, we examined their origin by lineage analysis in slices of embryonic human forebrain tissue.

The developmental period analysed ranged from 10 to 25 weeks of gestation, which corresponds to the major wave of generation and migration of cortical neurons in the human brain ${ }^{22}$. To determine the pattern of migration of GABAergic neurons, we first immunostained cortical sections for GABA, Dlx1/2 homeodomain proteins and neuron-specific $\beta$-tubulin/TuJ1 antigen and analysed immunoreactive cells with migratory morphologies. We found that immunoreactivity for all three antigens (GABA/Dlx/ TuJ1) defined a population of mostly non-radially oriented migratory-like neurons within the ventricular and subventricular zone (VZ/SVZ) (Fig. 1a, b). Numerous migratory-like, radially oriented GABA/Dlx-immunoreactive cells were present in the intermediate zone (IZ), situated between the proliferative VZ/ SVZ and the cortical plate (Fig. 1c).

We next performed a direct analysis of cell migration in organotypic slices of the embryonic cerebral cortical wall. Crystals of DiI were placed into the VZ/SVZ and the distribution of migrating cells examined after $36-48$ hours in culture (Fig. 1d, e). At 10 weeks of gestation $95 \%$ of labelled cells analysed ( $n=180$ from 21 slices) migrated radially in the VZ/SVZ and the IZ. From 13 weeks of gestation the incidence of non-radially migrating cells and their proportion in the total population of migrating cells significantly increased, although we did not observe an overall increase in the magnitude of cell migration (that is, in the number of cells observed away from a DiI crystal per slice). Thus, in the VZ/SVZ of specimens that were 14 and 24 weeks old, $60-65 \%$ of labelled cells analysed ( $n=255$ cells from 30 slices at 14 weeks of gestation; $n=320$ cells from 28 slices at 24 weeks of gestation) migrated non-radially, even though in the IZ, around $75 \%$ of labelled cells analysed ( $n=150$ per fetal stage) migrated radially (Fig. 1d, e).

To demonstrate directly non-radial migration of neuronal cells within the VZ/SVZ, we designed a DNA construct encoding a yellow fluorescent protein (EYFP) under the control of neuron-specific Ta1 promoter (Fig. 1f). This construct labels neurons exclusively (Fig. $1 \mathrm{~g}, \mathrm{~h}$ ) and enables time-lapse analysis of their migration (Fig. 1i). We have observed an extensive non-radial migration of EYFPpositive cells in the VZ/SVZ of neocortical slice cultures (for example, see Fig. 1i). Electron microscopic analysis confirmed the existence of a network of non-radially migrating TuJ1/GABApositive neurons within the VZ/SVZ and suggested that these cells rely on neurophilic-homotypic guidance (Fig. 1j). Moreover, when neocortical VZ/SVZ explants where cultured in Matrigel, a massive neurophilic migration of TuJ1/GABA/Dlx-positive neurons was noted out of the explants (Fig. 1k, 1), whereas only gliophilic migration along radial glial fibres occurred out of IZ explants (Fig. $1 \mathrm{~m}$ ). Thus, both morphological and in vitro data together indicate that GABAergic neurons migrate in non-radial, neurophilic fashion within the VZ/SVZ, but switch to radial, gliophilic mode in the IZ, after exiting the VZ/SVZ.

We observed in the developing human cerebral wall a distinct subpopulation of GABAergic neurons, that in addition to Dlx1/2 also expresses Mash1 (Fig. 2a-d). Thus, at 25 weeks of gestation, $65.1 \pm 1.6 \%$ of GABA/Dlx1/2-positive cortical plate neurons analysed ( $n=600$ ) expressed Mash1. Furthermore, about two-thirds of migrating GABAergic neurons in the VZ/SVZ analysed $(n=600)$, belonged to this class $(70.2 \pm 3.3 \%$ at 14 weeks and $61.8 \pm 3.0 \%$ at 24 weeks of gestation). Strikingly, our analysis also revealed that $8 \%$ of M-phase VZ progenitors and $85 \%$ of M-phase SVZ progenitors analysed ( $n=180$ for VZ and 240 for SVZ) expressed Mash1 (Fig. $2 \mathrm{e}-\mathrm{g}$ ), thus strongly suggesting that Mash1-positive GABAergic neurons originate in the dorsal forebrain proliferative zone.

To test this possibility, we exposed slices of the embryonic human cerebral cortex to a replication-incompetent retrovirus encoding green fluorescent protein (GFP) under a constitutive promoter. The slices did not contain the GE, thus excluding the possibility of labelling cortical migrating cells that may originate in the GE. In the VZ/SVZ of the dorsal telencephalon, GFP-positive clones were observed $18-24 \mathrm{~h}$ after the infection and their position periodically recorded every 2-6 h for two to three days (or every 20-30 min for a few hours in some cases). The infections resulted in one or two 